\title{
A good way to model phenomenas in agriculture research
}

\section{Opinion}

Knowledge representation and reasoning under uncertainty in natural resource management have a growing interest in Science and technology. There is a rising interest for a probabilistic methodology as tool for ecological and agronomic modelling which can help in the decision-making. This methodology has been discovered a long time ago by reverend Bayes Bayes ${ }^{1}$ and used for the first time by the mathematician Laplace.

\section{What is a bayesian network ?}

i. It is a graphical, intuitive model, direct representation of the problem to solve

ii. It is constituted of variables and dependency relations (interactions), based on inference.

Inference is the ability to deduce the value of a variable knowing that the variables that condition it are in a given state. For example in clinical medicine: the probability for an individual to develop a serious lung disease knowing that he is a smoker or not. So, bayesian networks are interesting formalisms to represent uncertain knowledge in artificial intelligence. The probabilities associated with each variable of a Bayesian network comes either from expert knowledge or from the density of probability of experimental data. In vine growing we proposed a probabilistic model able to assess the quality of a vineyard. Abbal et al. ${ }^{2}$

A vineyard is a complex system. Our vineyard model (Figure 1) takes into account the geographical area, chemical and physical properties of the soil, agronomic variables and water resources. The experts chose to consider the variables belonging to these groups because each of these families includes factors limiting the cultivation
Volume 8 Issue I - 2018

\author{
Philippe Abbal \\ Institute National de la Recherche Agronomique, Science for \\ Enology, France
}

Correspondence: Philippe Abbal, Institute National de la Recherche Agronomique, UMR I083, Science for Enology, bat. 28, 2 Place Viala, 34060 Montpellier, France, Email philippe.abbal@inra.fr

Received: December 19, 2017 | Published: January 12, 2018

of the vine Carbonneau et al. ${ }^{3}$ This version is based on a previous model that was designed to assess the quality of large wine areas Abbal et al. ${ }^{2}$ In this new version, we added the local drought index or soil water reservoir (W0), the vineyard balance, the vigor, the yield and the kind of wine expected (early, regular, long conservation). Furthermore, vine varieties have been ranked into four quality classes taking into account Huglin index associated with the level of their ability to elaborate top wines. This model assessing the potential quality of a vineyard is a real decision-making tool for a professional who wishes to quantify the qualitative potential of a vineyard. We tested the model succesfully in existing south of France vineyards. The appropriation of this model at the level of the wine sector will provide solutions for most current and future problems and at the same time will help to improve the model itself by testing it in as many situations as possible. This model is an example that could be used as a pattern in other scientific fields.

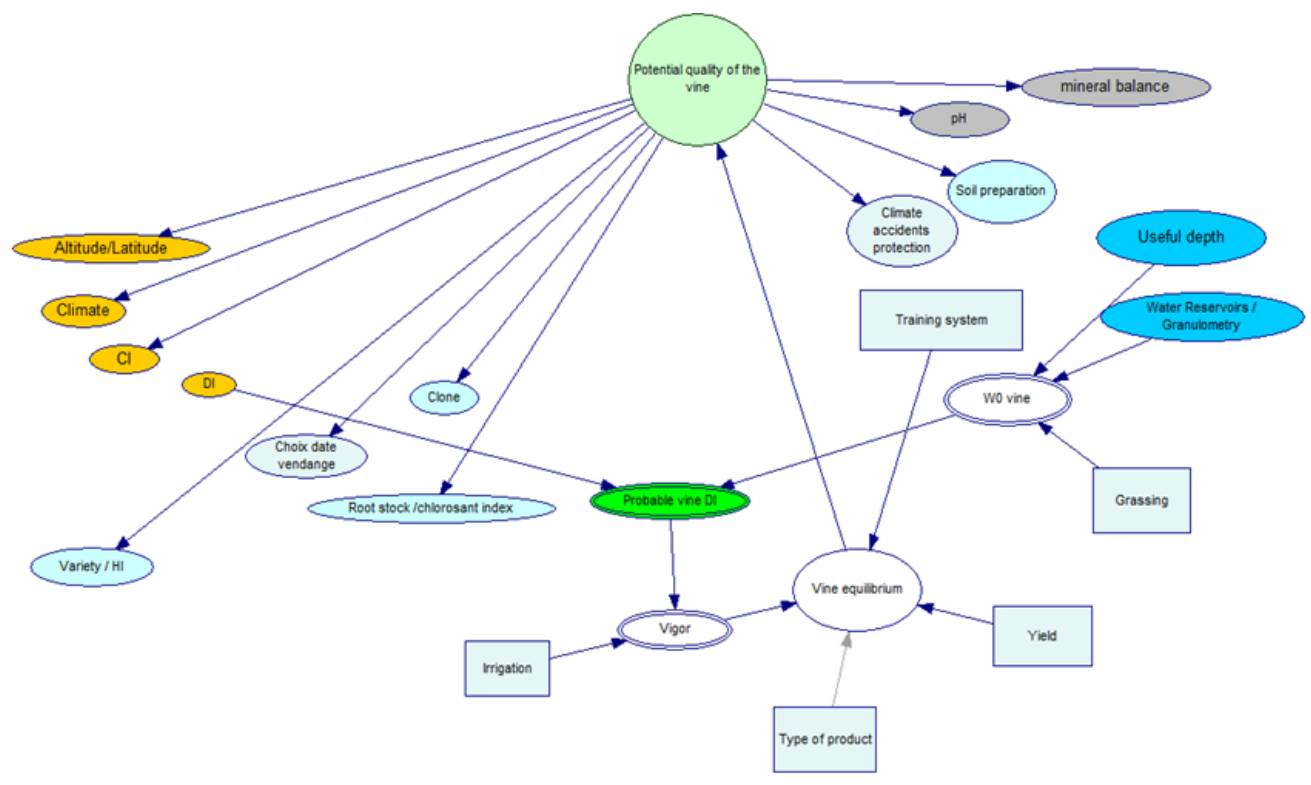

Figure I Model of the potential quality of a vineyard. 


\section{Acknowledgements}

This research was fully supported by National Institute of Agronomic Research (INRA, France).

I would like to thank Professor Alain Carbonneau, professor at Sup Agro Montpellier (France), Hernan Ojeda, manager of Inra Gruissan research station (France) and Nicolas Saurin engineer at Inra Gruissan (France). I am thankful to my colleagues Cedric Baudrit, a scientist at INRA Bordeaux (France), to Pierre Henri Wuillemin, Assistant Professor at Paris 6 University(France), and to John Guiver, a computer engineer from the machine learning group of Microsoft Research in Cambridge UK, for their help and advice concerning Bayesian reasoning.

\section{Conflict of interest}

I, Philippe Abbal, certify that I have no affiliations with or involvement in any organization or entity with any financial interest, or non-financial interest in the subject matter or materials discussed in this manuscript.

\section{References}

1. Bayes Th. An essay towards solving a problem in the doctrine of chances. Philosophical Transactions of the Royal Society of London. 1763;53(1763):370-418.

2. Abbal P, Sablayrolles Jm, Matzner Lober E, et al. A decision support for vine growers based on a Bayesian Network. Journal Of Agricultural Biological And Environmental Statistics. 2016;21(1):131-151.

3. Carbonneau A, Deloire A, Jaillard B. La Vigne: physiologie, terroir, culture. Paris, Dunod, France; 2007. 464 p. 